$$
\begin{aligned}
& \Delta s_{b}=\frac{R x^{\prime}}{\beta_{s}} \ln \frac{1}{1-\frac{n_{2} \beta_{s}}{n_{1}+n n_{2}}}-\frac{R x^{\prime}}{x n} \ln \frac{1}{1-\frac{n_{2} x n}{n_{1}+n n_{2}}} \\
& \Delta s_{b}=\frac{R x^{\prime}}{\varkappa n} \ln \left[1-\frac{n_{2} \varkappa n}{n_{1}+n n_{2}}\right]-\frac{R x^{\prime}}{\beta_{s}}\left[\ln 1-\frac{n_{2} \beta_{s}}{n_{1}+n n_{2}}\right] .
\end{aligned}
$$

Entwickeln wir den Logarithmus in eine Reihe bis zum 2. Glied, so ergibt sich

$$
\begin{aligned}
\Delta s_{b} & =-R x^{\prime} \frac{n_{2}}{n_{1}+n n_{2}}+R x^{\prime} \frac{n_{2}}{n_{1}+n n_{2}}-\frac{R x^{\prime}}{2} \frac{n_{2}^{2} \varkappa n}{\left(n_{1}+n n_{2}\right)^{2}}+\frac{R x^{\prime}}{2} \frac{n_{2}^{2} \beta_{s}}{\left(n_{1}+n n_{2}\right)^{2}} \\
& =\frac{R x^{\prime}}{2} \frac{n_{2}^{2}}{\left(n_{1}+n n_{2}\right)^{2}}\left(\beta_{s}-\varkappa n\right) .
\end{aligned}
$$

Bei geringen Konzentrationen ist $n n_{2} \ll n_{1}$, und wir erhalten schließlich

$$
\Delta s_{b}=R\left(\frac{n_{2}}{n_{1}}\right)^{2} \frac{x^{\prime}\left(\beta_{s}-\varkappa n\right)}{2} .
$$

\title{
Ein Beitrag zum Eiszeitproblem
}

\section{Von Kurt Himpel}

Aus dem Physikalischen Institut der Universität Frankfurt a. M.

(Z. Naturforschg. 2 a, 419-427 [1947]; eingegangen am 1. April 1947)

Im ersten Teil werden kurz die entscheidenden Schwierigkeiten der beiden bekanntesten Versuche zur Erklärung der Eiszeiten, und zwar der Pol- bzw. Kontinentalverschiebungen (Epeirophorese) und der sog. astronomischen Theorie (Schwankungen der Schiefe der Ekliptik und der Erdbahnelemente) aufgezeigt. - Im zweiten Teil wird wahrscheinlich gemacht, daß eine schon 1921 vorgetragene Hypothese von S h a p le y, rach der die Sonne in der diluvialen Eiszeit eine von kosmischen Dunkelwolken verursachte schwache Veränderlichkeit ihrer Strahlung zeigte, nach neueren, in erster Linie astrophysikalischen Forschungsergebnissen eine brauchbare Erklärung zu liefern imstande ist, aus folgenden drei Gründen:

1. In allen dichteren kosmischen Dunkelwolken findet man unregelmäßige veränderliche Sterne eines ganz besonderen Typus; im Gegensatz $\mathrm{zu}$ fast allen übrigen Typen veränderlicher Sterne handelt es sich bei diesen Sternen um Zwergsterne, wie die Sonne oder noch schwächer.

2. Unser Sonnensystem befindet sich gegenwärtig innerhalb dunkler kosmischer Materie, und es ist höchstwahrscheinlich, daß es in naher Vergangenheit auch dichtere Teile von solchen Dunkelwolken durchquert hat.

3. Die Form der Dunkelwolken, ihre armförmigen, zirrus- oder zirrostratusähnlichen Anordnungen und Verästelungen lassen eine zwanglose Erklärung der mehrfachen Wiederholung der Eiszeiten zu; es wird hier also nicht nur die Eiszeit, sondern aus der gleichen Hauptursache auch deren Gliederung erklärt, was sonst nicht gelungen zu sein scheint. -

Die Mitwirkung anderer, in erster Linie astronomischer und geographischer Faktoren, letztere insbesondere in der älteren Erdgeschichte, ist durchaus möglich.

$\mathrm{V}$ or dreißig bis vierzig Jahren sah man der Lösung des Eiszeitproblems hoffnungsfreudiger entgegen als heute. Damals... erschien eine Hypothese über die Ursachen der Eiszeit nach der anderen... Heute ist man im allgemeinen bescheidener geworden und beschäftigt sich wieder mehr mit den Grundtatsachen ..." So lesen wir in dem ausgezeichneten Referat über den Stand des Problems bei Schw ar z b a c h ${ }^{1}$. Die Ursache für einen gewissen Pessimismus ist aber wohl nicht

1 M. Schwa r zbach, Das Problem der Eiszeit vom geologischen Standpunkt, Verlag F. Hirt, Breslau 1941. 
allein in einer Vermehrung des Beobachtungsmaterials zu sehen, dem die bisherigen Hypothesen nicht mehr gewachsen wären, sondern vor allem darin, daß die beiden großen Versuche, denen man in weitesten Kreisen eine Lösung des Problems zugetraut hatte, nicht zum Ziel gelangt sind: die Hypothese der Pol- und Kontinentalverschiebungen einerseits, die sog. astronomische Theorie andrerseits. Es mußte jedenfalls auffallen, daß unter den Forschern, die diese Hypothesen überhaupt ablehnen, gerade auch solche sind, denen unser Wissen über die Eiszeit am meisten. verdankt. Einen noch besseren Hinweis bildet der Umstand, daß neuerdings auch von maßgebender Seite ganz neue Gedankengänge zum Vortrag gebracht worden sind. So mag vorwegnehmend Simpson ${ }^{2}$ erwähnt werden, der unter Verwendung moderner klimatologischer Ergebnisse spontane Variabilität der Sonne als Ursache der Eiszeit annimmt, wogegen, wenn auch unter sich gänzlich verschieden, Ki r s h ${ }^{3}$ und Wag ne r Schwankungen im radioaktiven Wärmestrom des Erdinnern als primäre Ursache betrachten.

Bevor ein ganz neuartiges astrophysikalisches Ergebnis mit dem Problem in Verbindung gebracht werden wird, möge noch einmal ganz kurz gezeigt werden, an welchen Hauptpunkten des Beobachtungsmaterials die oben erwähnten $\mathrm{Hy}$ pothesen scheitern mußten.

Pol-und Kontinentalverschiebungen (Epeirophorese)

Ich behandle hier beides zusammen, da wir in Ermangelung eines absoluten Bezugssystemes eine exakte Trennung nicht vornehmen können, eine solche auch paläoklimatologisch belanglos ist.

Neben dem allgemein bekannt gewordenen Versuch von W. Köppen u. A. Wegener ${ }^{5}$ sind noch andere vorgeschlagen worden, von denen besondere Erwähnung der von Gutenberg ${ }^{6}$ sowie der, speziell für das Permokarbon ausgearbeitete, von $\mathrm{Salomon}-\mathrm{Calvi}^{7}$ verdienen.

2 C. G. Simp s on, Quart. J. Roy. Meteorol. Soc. 60, 425 [1934] ; v. Fi c k e r, Meteorol. Z. 52, 165, 268 [1935].

3 G. K i rsch, Geologie und Radioaktivität, Berlin 1928; Geomechanik, Leipzig 1938.

${ }^{4}$ A. Wa g n e r, Klimaänderungen und Klimaschwan. kungen, Die Wissenschaft 92 [1940].

${ }^{5}$ Köppen-Wegener, Die Klimate der geologischen Vorzeit 1924; Ergänzung 1940.
Natürlich sind, vor allem solange man sich rein empirisch leiten läßt, auch andere Rekonstruktionen möglich.

Auf die theoretischen Schwierigkeiten, insbesondere die dynamischen, soll hier nicht eingegangen werden. Es ist ja längst bekannt, daß die rechnerisch gut erfåbbaren Kräfte der Westdrift und Polflucht größenordnungsmäßig um einen Faktor $10^{6}$ bzw. $10^{12}$ zu klein sind, um die Kontinente (widerstandslos) zu verschieben bzw. um durch Kontinentalverschiebungen ganze Gebirgszüge aufzubauen. Man müßte jedenfalls auf Unterströmungen zurückgreifen, denn ,,jedenfalls stehen uns in der säkularflüssigen $Z_{\text {one unter den }}$ Kontinenten Kräfte von ganz anderer Größenordnung zur Verfügung als die von We gen er angenommenen" (S a l o m o n, 1. c. ${ }^{7}$ S. 143). Es fragt sich allerdings, ob die thermodynamische Seite jener Deduktionen wirklich in Ordnung ist, worauf $\mathrm{Schw}$ inner ${ }^{8}$ kritisch eingegangen ist.

Einen Einwand von ganz anderem Gewicht sehe ich in der Tatsache, daß wir für die WürmEiszeit mit Sicherheit, für die älteren diluvialen Eiszeiten mit großer Wahrscheinlichkeit beweisen können, daß nur eine Pol-Lage zu ihnen paßt und das ist die jetzige (vergl. Klute ${ }^{9}$ )!

Weiterhin aber sollten bei Pol- oder Kontinentalverschiebungen in der Eiszeit solche Gebiete auffindbar sein, deren Klima wärmer als heute gewesen wäre. Das ist nirgends der Fall! Vielmehr erscheint uns heute die Würm-Eiszeit als eine den ganzen Planeten gleichzeitig betreffende Erscheinung, und für die älteren diluvialen Eiszeiten gilt zweifellos das gleiche.

Ist es immerhin bedenklich, daß Pol- und Kontinentalverschiebungen gerade dort versagen, wo ${ }^{*}$ wir einer Klärung vordringlich bedürfen, so dürfte nach neueren Forschungen Ähnliches auch für die warmen Klimate des Tertiärs und Mesozoikums gelten. Dem Gewinn auf der Nordhalbkugel steht ein gleich großes Defizit auf der Südhalbkugel gegenüber, wo man den Pol in gemäßigte oder subtropische Lebewelten hinein-

${ }_{6}^{6}$ B. Gut e n be r g, Gerlands Beitr. Geophysik 16, $239 ; 18,281$ [1927].

7 W. Salomon-Calvi, Die permokarbonischen Eiszeiten, Leipzig 1933.

8 R. Schwinner, ,Über den Wärmehaushalt des Erdballes“, Gerlands Beitr. Geophysik 58, Heft 3/4 [1942].

${ }^{9}$ F. Klute, ,Eiszeit und Klima“ in Frankf. Geogr. Hefte 11, 56 [1937]. 
bekäme, wie es $\mathrm{Kerner-M}$ arilaun ${ }^{10}$ und $\mathrm{Hi} \mathrm{m} \mathrm{p} \mathrm{e}{ }^{11}$ näher ausgeführt haben.

In noch älteren Formationen bilden die permokarbonischen Eiszeiten allerdings ein solches Bild, daß man ohne eine weitgehende Epeirophorese nicht auszukommen scheint. Meines Erachtens könnte man tatsächlich sich auf eine solche festlegen, würden nicht die noch älteren (früh- bzw. präkambrischen und huronischen) Eiszeiten wieder mehr für eine der jetzigen gleiche Lage des Poles (ich denke in erster Linie an den Nordpol) sprechen. We g e n e rs Vorgehen, die Erdgeschichte sozusagen erst im Oberkarbon beginnen zu lassen, mochte damals noch angängig sein, heute ,kommt auf jeden Fall in die Entwicklungslinie ein Knick, welchen der Geologe ablehnen muß" (Schwinner ${ }^{12}$, S. 285).

Worauf mich Hr. Salomon (briefl. Mitt. 1938) besonders hinwies, wäre der rätselhafte Umstand, daß - was Wegener damals nicht wissen konnte - wir im Permokarbon außerdem mit einer warmen Antarktis zu rechnen haben, was Salomon mit Recht als ,den springenden Punkt des Problems" ansieht. Ist Epeirophorese wirklich die gesuchte Erklärung, dann kommen wir jedenfalls zu dem bizarren Bild, daß damals Antarktika in. niederen Breiten gelegen haben müßte und erst im Quartär an den Südpol wanderte! Dies nimmt Sal o m o n ${ }^{7}$ (S.128) auch tatsächlich an; eine Außerung dazu ist mir nicht bekannt geworden. Darum lehnt er auch Wege ners „Polflucht" ab (S. 143).

Recht naheliegend ist in solchem Falle allerdings der Gedanke, eine temporär größere Schiefe der Ekliptik könnte die Phänomene der permischen Eiszeit verursacht haben oder wenigstens für die eigenartige Verteilung der Eisspuren verantwortlich gemacht werden; aber die Ursache für solche Schwankungen der Schiefe, die mindestens zeitweise $\sim 40^{\circ}$ erreicht haben müßte, wäre schwer anzugeben (1. c. ${ }^{13}$, S. 42$)$ !

Da sich alle Paläoklimatologen darüber einig sind, daß die bei weitem bedeutendste Frage ihres Forschungsgebietes die nach der Hauptursache der diluvialen, weltweiten Eiszeiten ist, hier aber der Beitrag der besprochenen Theoriengruppen

${ }^{10}$ F. Kerner-Marila un, Z. dtsch. geol. Ges. 86, Heft 2 [1934].

11 K. Hi m pe l, Meteorol. Z. 55, 256 [1938].

12 R. Schwinner, Lehrb. d. physikal. Geologie I 1936. jedenfalls sehr bescheiden ist, können Pol- und Kontinentalverschiebungen nur ein modifizierender, nicht aber ein dominierender Faktor im Vorweltklima gewesen sein.

Astronomische Theorieder Klima$\mathrm{schw}$ ankungen

Viel mehr Anhänger als die Epeirophorese hat aber die sog. astronomische Theorie der Klimaschwankungen gefunden, derzufolge die schwankenden (und von der theoretischen Astronomie auf Jahrhunderttausende verfolgbaren) Erdbahnelemente Klimaschwankungen hervorrufen sollten ${ }^{14}$.

Vorwegnehmend darf allerdings klärend festgestellt werden, daß sich alle, auch die überzeugtesten Verfechter dieser Hypothese restlos darüber im klaren sind, daß ihre Hypothese nur einen Teil des Problems zu bewältigen imstande ist, und zwar die Gliederung der Eiszeit; Anfang und Ende der Eiszeit werden durch diese Schwankungen nicht erklärt. Die Tatsache jedoch, daß vielfach, vor allem in populären Darstellungen, die astronomische Theorie als die Erklärung hingestellt wird, kann m. E. nur psychologisch begriffen werden: Die Triumphe der theoretischen Astronomie seit Newtons Tagen beschatteten diese Hypothese, die für sich das stolze Wort von der „astronomischen Genauigkeit“ in Anspruch nehmen wollte. Aber wir handeln mit Edelsteinen in der Wüste..., denn der gewaltige Trugschluß ist der, daß die Geologie ein fertiges Schema vorlegen könne, das wir sozusagen nur mit Jahreszahlen zu beschriften hätten! Nein, die absoluten, d. h. von Theorien über die Eiszeit unabhängigen Altersbestimmungen des Quartärs sind viel zu unsicher, um das Milankovitehsche Schema daran prüfen zu können! Wir erhalten absolute Werte entweder durch die bekannte radioaktive Methode und kommen so für die Grenze QuartärPliozän zu 0,6 bis $1 \cdot 10^{6}$ Jahren. Oder man geht von der kleinsten geologischen Größe, der Jahresablagerung (z. B. d e G e e r - Methode), extrapolatorisch weiter und kommt so für die Günz-Eis-

${ }_{13} \mathrm{~K}$. Himpel, Erdgeschichte und Kosmogonie, Leipzig 1940.

${ }^{14}$ Von dem umfangreichen Schrifttum sei nur zitiert: Milankovit ch, Hdb. d. Klimatologie I, Tl. A, 1930; Hdb. d. Geophysik 9, Lief. 3, 1938; Meteorol. Z. 55, 76 [1938]; R. Spitale r, Die Bestrahlung der Erde usw., Abh. Dtsch. Ges. Wiss. Prag [1940]. Vergl. auch Fußnote 19. 
zeit zu rund $0,5 \cdot 10^{6}$ Jahren zurück, also größenordnungsmäßig in Übereinstimmung mit der radioaktiven Zeitskala.

Daß von einer „Bestätigung“ eines astronomischen Schemas durch die Geologie nicht einmal entfernt die Rede sein kann, das beweist allein die kuriose Tatsache, daß neben dem Milankovitchschen noch das Spitalersche Schema ${ }^{14}$ besteht, beide äußerlich ähnlich, nur in der Zeitskala um gut das Doppelte auseinander!! Ohne irgendwie auf die Ursache der Diskrepanz eingehen zu wollen, sei nur die Bemerkung gestattet, daß man mit jedweder willkürlichen Annahme von Schiefe und Exzentrizität immer zu einem solch ,gezackten“ Schema von etwa der gleichen Intervalldauer kommen wird, und daß, weil die absolute geologische Zeitskala zu ungenau ist, man jedes solcher Schemata ,bestätigen“ könnte. Daraus ergibt sich:

a) Es ist für die Prüfung der astronomischen Theorie belanglos, ob man die Zuverlässigkeit der Zurückrechnung zugibt oder sie in Zweifel zieht (von astronomischer Seite hat sich völlig ablehnend $\mathrm{z}$. B. Z i n $\mathrm{n}$ e $\mathrm{r}^{15}$ ausgesprochen).

b) Die astronomische Hypothese hat vor anderen paläoklimatischen Hypothesen keinen Vorsprung zu beanspruchen. Die großen Bedenken gegen die Hypothese fasse ich in folgende vier Punkte in äußerster Kürze zusammen:

Einwand 1: Warum finden wir astronomische Schwankungen nur im Quartär klimatologisch belegt, obwohl doch früher gelegentlich viel geringere Sommerbestrahlungen vorkamen? Aber diesen seitens der Kritik nur allzu breitgetretenen Einwand könnte man noch am leichtesten entkräften, und zwar damit, daß durch eine noch zu nominierende Hauptursache die Strahlungskurve entsprechend "herabgedrückt" worden sei, bzw. daß im eisfreien Polarklima die klimatologische Auswirkung astronomischer Schwankungen anders sei, eventuell sogar konträr, was Himpel ${ }^{13}$ zur Diskussion stellte.

Einwand 2: Im Karbon-Perm finden wir, vor allem in Australien, bis zu 6 große Eisvorstöße, getrennt durch Interglaziale von der Größenordnung 3 bis $5 \cdot 10^{6}$ Jahren, also das 20-50-fache der quartären Intervalle. Sow ohl Sal o mon ${ }^{7}$ (S.142) als auch Schwinner ${ }^{12}$ (S. 298) haben daher die Anwendbarkeit der Milankovitchschen Schwankungen für das Permokarbon mit $B e-$ stimmtheit abgelehnt!
Da ein solches Vorgehen aber nur ein Rätsel lösen, dafür aber drei neue aufwerfen würde, hat $\mathrm{Hi}$ m e $\mathbf{l}^{11}$ den Versuch gemacht, aufzufinden, unter welchen Bedingungen die astronomische Theorie auch für das Permokarbon brauchbar wäre. Es zeigte sich, daß dazu die Erdbahn noch etwas kreisförmiger und die Schiefe etwas kleiner als heute gewesen sein müßten, beides überraschenderweise Dinge, die von der Kosmogonie aufs stärkste befürwortet werden. Der Umstand, daß von maßgebender astronomischer Seite sofort auf diese Arbeit hingewiesen ${ }^{16}$ wurde, beweist die Wichtigkeit der Problemstellung. Es ist verwunderlich, daß von seiten der Vertreter der astronomischen Theorie dazu noch nicht Stellung genommen wurde, obwohl doch an dieser Diskrepanz, falls sie nicht aufgeklärt werden könnte, die astronomische Theorie scheitern müßte.

Einwand 3: Meist heißt es, daß die Erdbahnelemente nicht die Gesamtstrahlung, sondern nur deren jahreszeitliche Verteilung beeinflussen. Aber das ist nur so lange zutreffend, als man den in die Strahlungssumme eingehenden Faktor $1 / \sqrt{1-e^{2}}$ auch wirklich gleich 1 setzen kann. Ging, wie im Quartär, die Exzentrizität bis 0,06 herauf, dann ergibt sich bereits ein Mehr von $2 \%$. Ist das vernachlässigbar? Alle Paläoklimatologen sind sich darüber einig, daß schon ein Plus bzw. Minus von der Größenordnung 1-2\% weltweite klimatische Einflüsse hätte, falls es nur genügend lange wirkt. Und hier haben wir ein Mehr über mindestens 20000 Jahre. Wenn andrerseits z. B. Spitaler ${ }^{14}$ als zusätzliche Strahlungsquelle die veränderliche Lage des Milchstraßengürtels zum Erdäquator (!) allen Ernstes diskutiert, oder wenn $\mathrm{Nölke}^{17}$ an zusätzliche Strahlung des ehedem näheren Erdmondes denkt, dann kann man den obigen Betrag nur sehr ernst nehmen, denn die geschilderten Effekte sind von der Größenordnung $10^{-8}$ der Sonnenstrahlung, also $1^{-5}$ des Exzentrizitätseffektes! Eiszeiten könnten demnach viel eher bei kleinen Exzentrizitäten plausibel gemacht werden, und das gilt erst recht für die Tropenvergletscherungen, da in den Tropen ein Mehr an Gesamtstrahlung die hier ohnehin weniger ins Gewicht fallenden jahreszeit-

15 E. Z in ner, Astron. Nachr. 249, 88 [1933].

${ }_{16}$ H. K o bo ld, Liter. Beiblatt zu den Astron. Nachr. 1938, S. 61.

17 F. Nölke. Astronomie und Geologie. Z. dtsch. geol. Ges. 93, 142 [1941]. 
lichen Differenzen klimatologisch weit übertreffen müßte! Tropenvergletscherungen könnten nach dieser Hypothese also allenfalls bei kleiner Exzentrizität und großer Schiefe verständlich gemacht werden, während für höhere Breiten die genau konträre Kombination allgemein gefordert wird. Die Würm-Vereisung war jedoch gleichzeitig auf der ganzen Erde!

Einwand 4: Mit der klimatologischen Grundlage der Hypothese steht es heute schlechter, als man anfänglich glaubte, insbesondere im Hinblick auf die bedeutsamen Ergebnisse von A. W a g n e ${ }^{4}$. Wagner, der, wie kaum ein anderer, gerade zur Beurteilung dieser Seite des Problems imstande sein dürfte, sprach sich denn auch an verschiedenen Stellen (S. 155/156) dafür aus, daß die älteren, auf Croll zurückgehenden klimatologischen Vorstellungen (Eiszeiten bei strengen Wintern) mindestens die gleiche Berechtigung haben wie die vor allem auf $\mathrm{K}$ ö p pen zurückgehende moderne Meinung (Eiszeit bei kühlen Sommern)! Die wichtige Tatsache, daß in der jetzigen großen Klimaschwankung gerade die Erscheinung, die man für Eiszeiten verantwortlich machen wollte, nämlich Abnahme der Jahresamplitude bei verstärkter Zirkulation, kräftigen Rückgang der Gletscher erzwang, ebenso wie die Regel, daß auf die großen Winter die Jahresmittel zu niedrig zu sein pflegen, sprechen aufs überzeugendste zugunsten der „veralteten“ Auffassung! Sich hier „umstellen“ zu wollen, würde den Zusammenbruch der astronomischen Hypothese bedeuten, deren Vertreter sich auf die neuere Vorstellung viel zu sehr festgelegt haben, und müßte ja außerdem das Glanzstück der Hypothese, das Milankovitchsche Schema, ad absurdum führen!!

Nach diesen schwerwiegenden Einwänden möchte ich mich dem Endurteil von Wagner anschließen:

„Der Einfluß der Strahlungskurve nach Spitale r bzw. Milankoviteh wird nicht angezweifelt, wenn auch nicht gesagt werden kann, wie groß dieser ist, vielleicht nicht einmal, in welchem Sinne er wirksam ist. Er mag gelegentlich eine sich entwickelnde Eiszeit begünstigt, ein anderes Mal ihr entgegengewirkt haben. Aber im Sinne der entwickelten Theorie käme der Strahlungskurve nur ein modifizierender, nicht aber ein maßgebender Einfluß zu“" (1. c. ${ }^{4}$ S. 175, Hervorhebungen durch mich, K. H.).
Die Rücksichtnahme auf die Bereitschaft nicht nur der Erdatmosphäre, sondern des gesamten paläogeographischen Erdbildes, kosmische Einflüsse sozusagen erst in der ihnen angemessenen klimatologischen Sprache zu interpretieren, ist m. E. viel zu selten anzutreffen; die psychologische Reaktion auf der Gegenseite, kosmische Einflüsse ganz zu negieren, ist so ohne weiteres verständlich, aber ebenso ungerechtfertigt, wie etwa einem kosmischen Phänomen zu allen Erdzeitaltern die gleiche klimatologische Wirkung zuschreiben zu wollen.

Fassen wir den ersten Hauptteil zusammen: Bei aller Anerkennung der großen Verdienste der beiden großen Hypothesengruppen um das Eiszeitproblem können wir uns doch des Eindrucks nicht erwehren, daß sie an das eigentliche Problem in seiner ganzen Größe nicht recht herangekommen sind.

Wenn nun die besprochenen Hypothesengruppen, besonders die astronomische, durch eine bessere ersetzt werden sollen, dann bleibt von vornherein nur folgende Möglichkeit: Die Hauptursache hat auch die Schwankungen erzeugt. Die Eiszeit wird also, wie es die Beobachtungen so nachdrücklich fordern, als ein einheitliches Phänomen $\mathrm{zu}$ interpretieren sein.

Im zweiten Hauptteil soll nun ein neuartiges astrophysikalisches Ergebnis zur Erklärung der diluvialen Eiszeiten herangezogen werden, von dem bewiesen werden wird, daß es nicht nur sämtliche Vorteile der astronomischen Hypothese ebenfalls besitzt, sondern auch, daß es stark genug ist, die Eiszeiten und ihre Wiederholung zwanglos zu erklären.

\section{Passage des Sonnensystems durch eine kosmische Dunkelwolke}

Als diejenige Hypothese, die unter allen kosmischen Hypothesen die größten Sympathien genießt, darf, in erster Linie infolge des geschickten Vortrages von F. Nölke ${ }^{18}$, die einer Passage des Sonnensystems durch eine kosmische Dunkelwolke gelten; sie ist neben den obigen beiden Hypothesen wohl auch die am meisten bekannt gewordene Eiszeittheorie überhaupt, die die älteren, etwas mystischen Theorien von ,kalten und warmen Gegenden des Weltenraumes" abzulösen geeignet schien.

18 F. Nölke, Zuerst vorgetragen 1908, sehr zahlr. Arb., zuletzt z. B. Forschg. u. Fortschr. 17, 309 [1941]. 
Betrachtet man sich einmal die wunderschönen Aufnahmen der bekannten Milchstraßenatlanten von $B$ a rnard oder von $R o ß$, ebenso unsere nur einen bescheidenen Eindruck vermittelnden Abb.1-2, so erkennt man, daß die gesunde Grundlage der Hypothese kaum bezweifelt werden kann. Auch von maßgebender Seite der astronomischen Theorie wird ihr durchaus Bedeutung beigemessen (vergl. Wundt ${ }^{19}$ ). Wenn sich die Hypothese nicht zwingend durchsetzen konnte, dann aus folgenden Gründen:

a) Die Dichten der Dunkelwolken, über die wir verhältnismäßig sichere Werte haben, liegen bei der Größenordnung $10^{-25} \mathrm{~g} / \mathrm{cm}^{3}$, in den dichtesten Teilen vielleicht $1-2$ Größenordnungen mehr. Aber $1^{-23} \mathrm{~g} / \mathrm{cm}^{3}$ wäre schon der alleroberste Wert, und nur an kleinen Stellen anzutreffen! Aber auch bei dieser Dichte läßt sich abschätzen, daß die Absorption auf dem Wege Erde-Sonne günstigstenfalls $10^{-9}$ der Sonnenstrahlung betrüge. Außerdem kämen hinzu Erhellung der Wolke und deren nächtliche Rückstrahlung sowie Erwärmung der Erdatmosphäre (ebenso wie auch der Sonne selber), alles minimale Beträge. Die exakte Durchrechnung ergab denn auch ein geringes Überwiegen der Erwärmung, aber, wie gesagt, um einige 10-9 $^{-9}$ Größenordnungen kleiner als die Sonnenstrahlen, während allein der Effekt einer größeren Exzentrizität der Erdbahn den $10^{6}$-fachen Betrag einer solch minimalen Wirkung ausmacht. Auch Nölkes Hinweis auf eine sich mit der Zeit akkumulierende chemische Beeinflussung der Erdatmosphäre überzeugt nicht, nachdem ausgedehnte Zeiten lebhaften Vulkanismus' Materialmengen an Gas und Staub in die Erdatmosphäre transportiert haben, die um wenigstens eine Größenordnung über der betrachteten liegen, und trotzdem dadurch (z. B. im Eozän) keine Eiszeit entstanden ist.

b) Die Annahme von Dunkelwolkenpassagen müsse erst postuliert werden, wogegen die astronomischen Elemente wirklich geschwankt haben ( W und t ${ }^{19}$ ).

c) Das Alternieren der Eiszeiten ist schwer glaubhaft $\mathrm{zu}$ machen ${ }^{19}$.

Ich werde nun beweisen, daß alle Einwände nicht nur entkräftet werden können, sondern im

19 W. Wundt, Sehr zahlr. Veröffentlichungen. Zur Dunkelwolken-Hypothese äußerte er sich z. B. in ,Aus der Heimat" 1938, 272. Letzte Veröff. Geol. Rdsch. [1944].
Gegenteil starke Seiten dieser Hypothese darstellen.

Schon um die Jahrhundertwende wurde in der Gegend der hellen und dunklen Nebelmassen im Orion eine ganze Anzahl besonderer veränderlicher Sterne entdeckt, und H. Sh a ple y ${ }^{20}$ brachte 1921 die Hypothese vor, daß die Sonne, als sie jene Gegenden des Weltraumes passierte, ebenfalls zur Veränderlichkeit angeregt worden sei, wodurch auf Erden die diluviale Eiszeit entstand. Ich werde beweisen, daß diese Hypothese durch die Ergebnisse der modernen Astrophysik in einer solchen Weise gestützt wird, daß sie verdient, in den Kreis ernster Diskussion einbezogen zu werden.

Daß diese Hypothese, obwohl ich sie in fast jeder Zusammenstellung von Eiszeittheorien zitiert finde, sich nicht durchsetzte, ja nicht einmal zu den wahrscheinlicheren Hypothesen gerechnet wurde, war sehr begreiflich, denn:

a) Wer wollte beweisen, daß die veränderlichen Sterne des Orionnebels nicht eine kosmogonisch zu erklärende Partikularität dieses Gebildes sind?

b) Der Orionnebel ist von uns 1600 Lichtjahre entfernt. Mit der bekannten Translationsgeschwindigkeit der Sonne von $19 \mathrm{~km} / \mathrm{sec}$ findet man, daß die Sonne jene Gegenden im Alttertiär durchquert hat, wogegen der die Würm-Vereisung erzeugende Nebel 1-2 Lichtjahre weg sein müßte!

c) Bei seiner Erwähnung der Shapleyschen Hypothese äußerte sich der als maßgebend anzusehende Eiszeitgeologe Coleman ${ }^{21}$ so, daß es nicht sicher sei, ob bei solcher solarer Veränderlichkeit nicht ein mildes Klima auf Erden ebensogut folgen könne wie eine Eiszeit.

Wie vorsichtig man sich auch in Amerika, trotz der hohen Autorität Shapleys, zu seiner Eiszeittheorie äußert, dafür ein Beleg aus einem drüben sehr bekannt gewordenen Buche ${ }^{22}$ (S.282). „It appears possible that at times of great ice ages the sun may have been in regions of space where existed enormous stretches of such material... Shapley has ventured the hypothesis that the presence of such material in the immediate neighbourhood of the sun may have directly

${ }^{20} \mathrm{H}$. Sh a pley, Note on a possible factor in changes of geological climate, J. Geology 29,501 [1921].

${ }^{21} \mathrm{I}$. C o l e m a n, Ice ages, recent and ancient, New York 1926.

${ }^{22}$ H. St et s o n, Earth, radio and the stars, New YorkLondon 1934. 


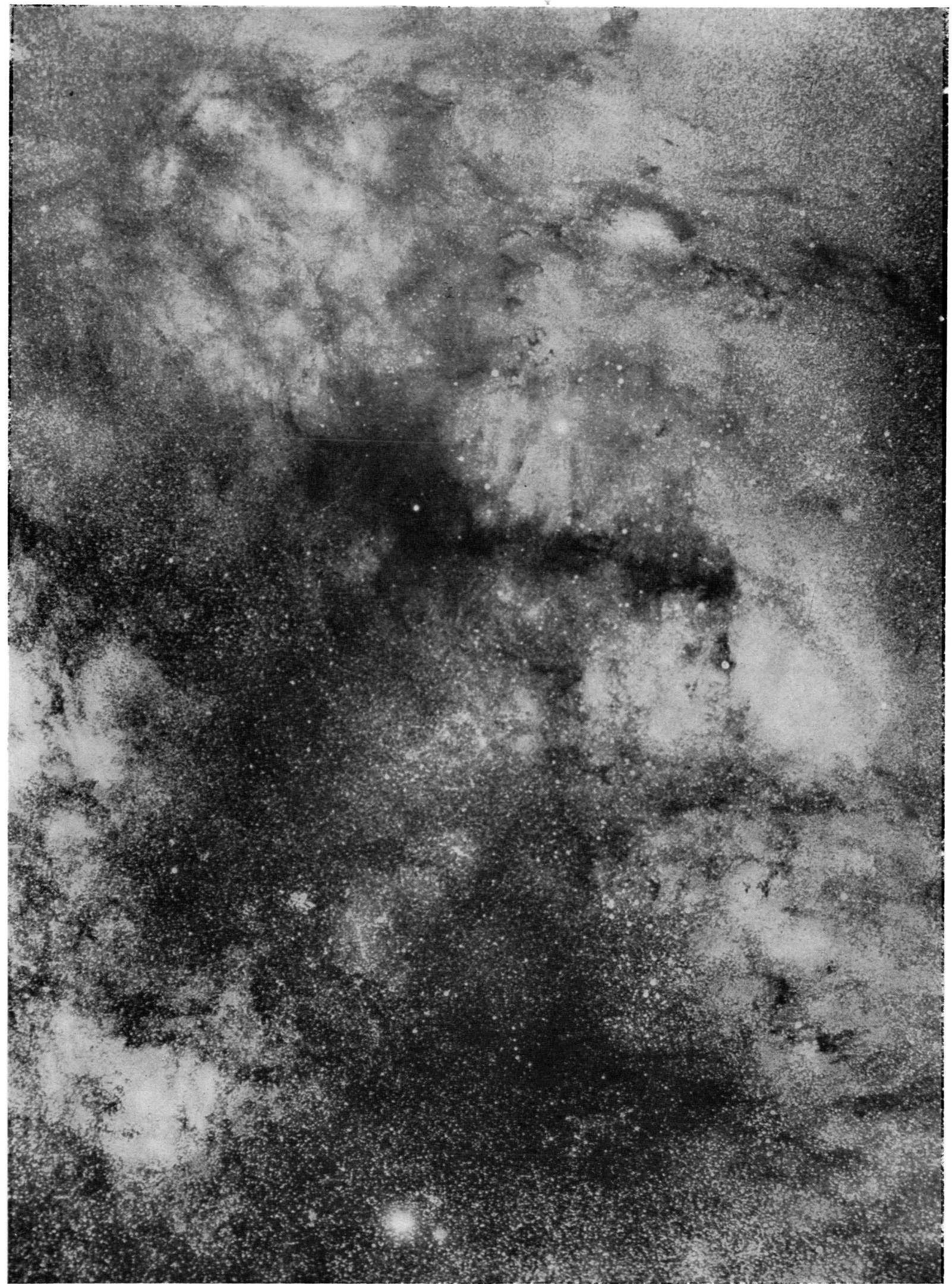

Abb. 1. Blick in das Zentrum des Milchstraßensystems. Sternbilder Schütze - Skorpion. (Kopien aus dem Milchstraßenatlas von F. E. R o \&, die mir Hr. H a i d r i c h, Wien, dankenswerterweise ausgeführt hat, da der Atlas in Deutschland nur in wenigen Exemplaren vorhanden ist.) Man beachte sowohl die gewaltigen als auch die zahlreichen kleineren Dunkelwolken und Verästelungen. 


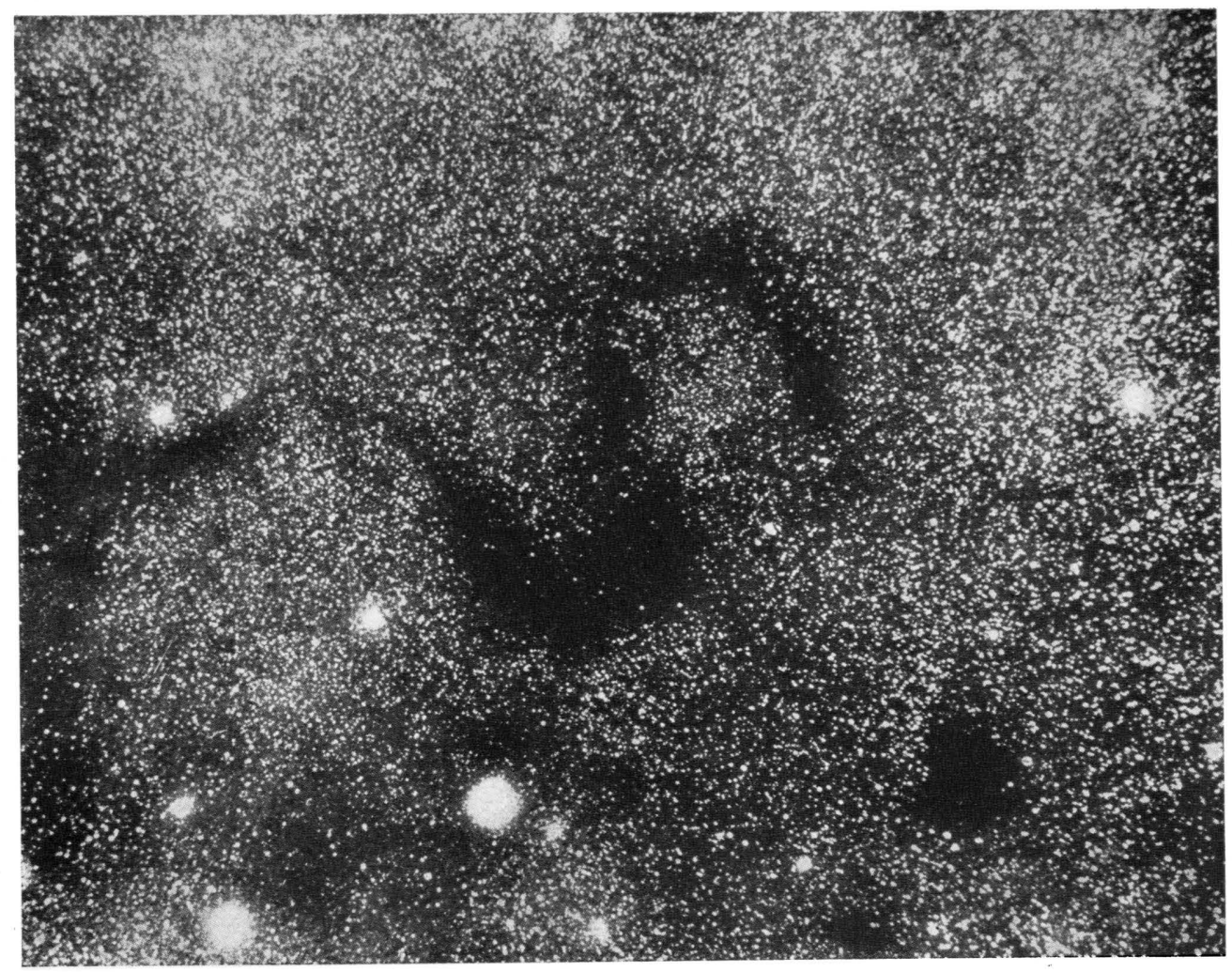

Abb. 2. Kleine, hufeisenförmige Dunkelwolke, aufgenommen mit dem 100-Zoller (Mt. Wilson). In Abb. 1 ist diese Wolke als winziges Gebilde zu erkennen und durch Pfeile an den oberen Rändern angezeigt. Im Sinne der entwickelten Hypothese käme eine solche Wolke als Ursache der diluvialen Eiszeiten in Betracht, während ein den großen Gebieten in Abb. 1 vergleichbares Wolkengebiet für die permokarbonische Eiszeit eine Erklärung liefern würde.

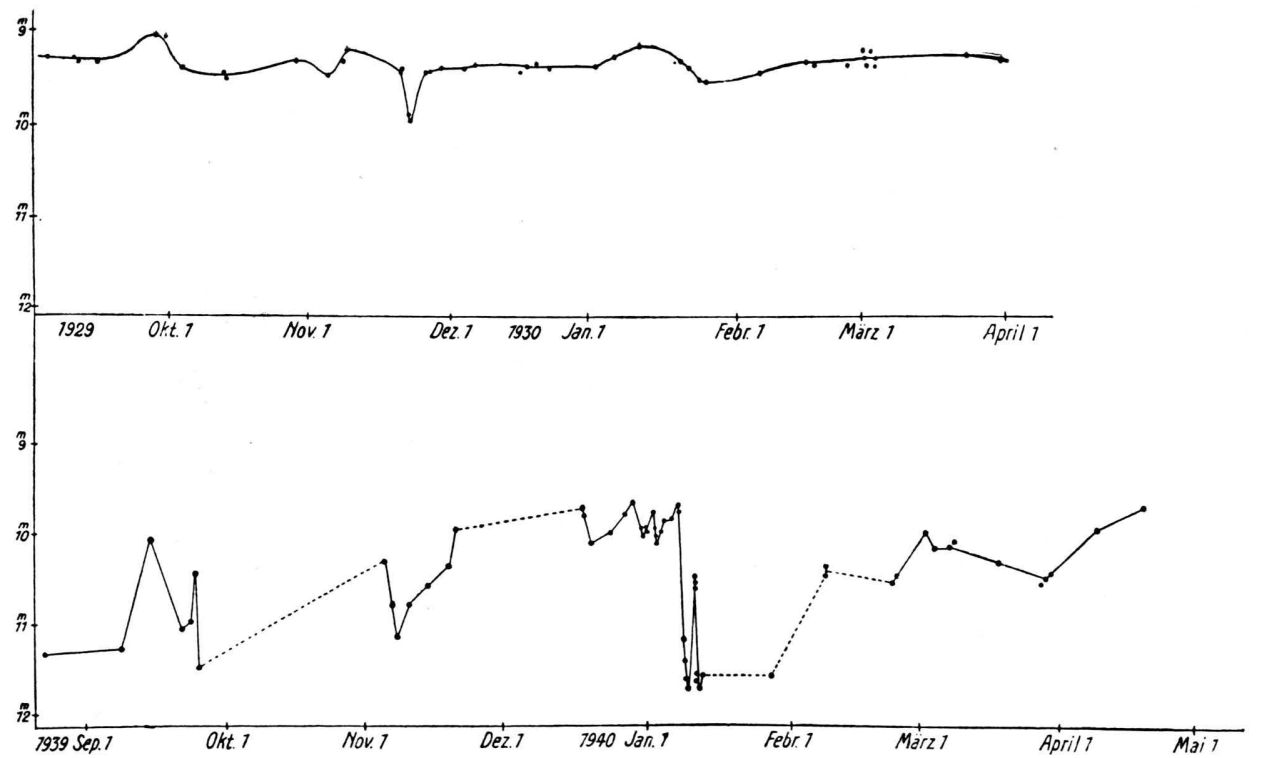

Abb. 3. Lichtkurve eines typischen Orionveränderlichen (BN Orionis) nach Beoh.d. Verf. (Aus Himmelswelt 1942.) 
affected the sun's output within geologic time." Der gegenwärtige Stand ist jedoch folgender:

Zuerst wohl von C. Payne und S. Gaposch$\mathrm{kin}^{23}$ klar herausgearbeitet, existieren in allen daraufhin untersuchten kosmischen Dunkelwolken solche veränderlichen Sterne, wie sie zuerst nur im Orionnebel bekannt waren. In ihrem neuen Buche schließen sich Campbell und Jacchia ${ }^{24}$ dem voll an. Himpel ${ }^{25}$ konnte dann unter Beibringung eines großen eigenen Materials die Behauptung aufstellen:

1. In allen Dunkelwolken existieren diese Orionveränderlichen, vom Nebel zu Veränderlichkeit angeregte normale Sterne (Satz von der Universalität der Orionveränderlichen).

2. Zahlenmäßig übertreffen diese Veränderlichen alle übrigen Gruppen veränderlicher Sterne.

Wie groß der tatsächliche Prozentsatz veränderlicher Sterne in einer Dunkelwolke ist, darüber können wir nur beim Orionnebel eine untere $\mathrm{Ab}$ schätzung machen. Für $\sim 15 \%$ der Sterne bis zur 15. Sterngröße wurde Veränderlichkeit gefunden. Der eigentümliche Lichtwechsel (manchmal jahrelang nur geringe Schwankungen) und vor allem die Tatsache, daß wir veränderliche Sterne bei so schwachen Sterngrößen erst dann entdecken können, wenn sie größere Schwankungen zeigen (mindestens 1/2 Größenklasse), läßt vermuten, daß die wirkliche Prozentzahl viel größer ist, ja jeder Stern in größerem oder kleinerem Umfange veränderlich wird!

Sieht man sich einmal eine typische Lichtkurve eines solchen Orionveränderlichen an (Abb.3), so

${ }^{23}$ C. u. S. Gaposchkin, Variable Stars, Harv. Obs. Monog. No. 5 [1938], Chapt. 11.

${ }_{24} \mathrm{Campbell-Jacchia,} \mathrm{The} \mathrm{story} \mathrm{of} \mathrm{variable}$ stars. Chicago 1941, Chapt. 7.

${ }^{25} \mathrm{~K}$. Himpel, Die seltenen Typen der veränd. Sterne, Festschr. für A. Pr ey (im Druck).

${ }_{26-27}$ W. B e c k e r, Fortschr. d. Astronomie Bd. I [1938] und "Sterne und Sternsysteme" [1942].

${ }_{28}$ C. H offmeister, S.-B. preuß. Akad. Wiss. Physik.-math. Kl. 18 [1936]; Die Meteore, Leipzig 1937.

${ }_{29} \mathrm{R}$. Schw in ner, Fußnote 12, S. $151 \mathrm{ff}$., und „Meteoriten und Geologie, Gerlands Beitr. Geophysik 16, 195 [1927].

30 Auch das Zodiakallicht, vielleicht in physischer Verbundenheit mit der Sonnenkorona, wäre am einfachsten durch die Dunkelwolke zu erklären. Der sog. Gegenschein ist vermutlich eine Meteoritenansammlung in der Nähe der Erde an einem der Lag r ang e. schen Librationspunkte. Die von $\mathrm{H}$ of $\mathrm{fme}$ is ter beschriebenen Leuchtstreifen sind Einbrüche kosmischen Staubes in die obersten Atmosphärenschichten. $\mathrm{H}$ of f meister vermutet sogar Beziehungen zu den meteorologischen Singularitäten. erkennt man, daß auf Zeiten, manchmal Jahre während, in denen der Stern in einer Art „Normallicht" ist, immer wieder Zeiten rascher Veränderlichkeit, meist bei unternormaler Strahlung, eintreten. Hinzu käme noch, auf die Sonne angewandt, eine sehr wahrscheinliche Zunahme der gesamten solaren Aktivität, die, wie die 11-jährige Fleckenperiode bezeugt, schon heute, wo die solare Veränderlichkeit unter $1-2 \%$ liegt, gelegentlich meteorologische Einflüsse hat!

Nach zahlreichen neueren Untersuchungen kann es als gesichert gelten, daß das Sonnensystem z. Zt. in unmittelbarer Nähe von Dunkelwolken sich befindet (vergl. Becker ${ }^{26,27}$ ). Vermutlich sind wir sogar innerhalb einer Dunkelwolke, was durch folgendes wahrscheinlich gemacht wird: Nach Hoffmeister ${ }^{28}$ dürfte das interstellare System der Kleinkörper, das sich uns als Meteore zu erkennen gibt, von einer Dunkelwolke herrühren, und dann hat Schwinner ${ }^{29,12}$ wahrscheinlich gemacht, daß Meteoriten in geologischer Zeit nicht gefallen sind, daß wir es vielmehr vermutlich mit einem durch das Eindringen in eine Dunkelwolke während der letzten Eiszeit verursachten Phänomen zu tun haben. Schließlich dürften auch (vergl. Nölke) die Kometen, vor allem im Hinblick auf ihre sicher erwiesene Kurzlebigkeit, Relikte des Eiszeitnebels sein ${ }^{\mathbf{3 0}}$.

Daß in unmittelbarer Nähe des Sonnensystems aber auch dichteste Dunkelwolkenteile vorkommen, solche also, die die in ihnen stehenden Sterne zu Veränderlichkeit bringen, beweist die ziemlich zuverlässige Entfernungsabschätzung bei dem in der Tauruswolke stehenden Orionveränderlichen UZ Tauri, der uns näher als 100 Lichtjahre steht $^{31,32}$.

${ }^{31}$ K. H i m p e l, UZ Tauri, Beob. Zirk. Astron. Nachr. 25, 38 [1943].

${ }_{32}$ Im Sinne der entwickelten Theorie besonders wichtig ist das Ergebnis einer Untersuchung von J. $\mathrm{H}$ o p man n über den uns benachbarten galaktischen Sternhaufen der Hyaden (Astron. Nachr. 269, 87-99 [1939]), dessen eigene Worte ich zitiere: „Die scheinbaren Helligkeiten der Hyaden-Sterne "sind durch interstellare Materie um 0,31 $\pm 0,07 \mathrm{~m}$. F. geschwächt, eine Bestätigung der Annahme von $\mathrm{H}$. M iu ll e r u. a., daß sich die Sonne selbst in einer solchen Wolke befindet, die sich für uns auf die Sternbilder Taurus und Ophiuchus projiziert... Der Vergleich der restlichen Abweichungen gegen die Normalkurve einerseits mit der Anzahl der schwachen. Sterne in der Umgebung von 16 Hyaden-Sternen andererseits ergibt Anzeichen dafür, daß diese Dunkelwolke ähnlich örtlich verschieden dicht ist, wie man es in zahlreichen anderen Fällen ermittelt hat." (Hervorhebungen durch mich. K. H.) 
Ein Blick auf unsere Abb. 1 u. 2 wirft auch Licht auf das Problem der mehrfachen Wiederholung der Eiszeit, und man könnte in den oben angeführten Milchstraßenatlanten hunderte solcher Beispiele finden: Die Dunkelwolken zeigen eine deutliche Tendenz zu armförmiger Verteilung, so etwa wie unsere irdischen Zirren. Die Dunkelwolken in Abb. 1 haben dabei eine Ausdehnung von mindestens 500 Lichtjahren, die viel kleinere der Abb. 2 ca. 30 Lichtjahre. Nehmen wir also an, die Sonne habe in der vergangenen Diluvialzeit eine dieser kleinen Wolke ähnliche durchquert, so wäre sie 3- oder 4-mal zu Veränderlichkeit angeregt worden, was rasche Eisvorstöße hervorrief. Im Permokarbon hingegen dürfte sie ein so ausgedehntes Gebiet wie etwa in Abb. 1 am oberen Rande (es sind die berühmten Wolken um o Ophiuchi) durchmessen haben, wodurch Eiszeit und Interglaziale viel länger wurden. Aber für eine kritische Bewertung der Hypothese möchte ich doch bitten, erst einmal in den genannten Atlanten einen Eindruck von der unerschöpflichen Mannigfaltigkeit der Dunkelwolken zu gewinnen, um zu sehen, daß obige Abbildungen nicht ad hoc herangezogen sind.

Es ist ungemein wahrscheinlich, daß das Sonnensystem, z. Zt. innerhalb Dunkelwolken stehend, in geologisch jüngster Zeit auch dichtere Teile solcher Wolken passiert hat. Dabei ist es, dem hohen Prozentsatz veränderlicher Sterne in solchen Wolken entsprechend, wiederum sehr wahrscheinlich, daß auch die Sonne in solchen Wolkenarmen veränderlich war, wodurch, der Struktur der Wolken entsprechend, mehrere Eiszeiten entstanden.

Gewiß, der Gedanke, daß solare Veränderlichkeit die Eiszeit verursachte, ist nicht neu, er ist ja auch so naheliegend ${ }^{33}$. Aber erst die Verknüpfung mit Dunkelwolken läßt den vernichtenden Einwand, ein solches Stadium von Veränderlichkeit müsse erst postuliert werden, überwinden! Die Spontanität des Auftretens sowie die mehrfache Wiederholung, die bei anderen Versuchen stets zu ad-hoc-Postulaten zwingen mußte, erklärt sich völlig selbstverständlich.

Das enorm schwierige Problem, wieso die kosmisch bedingte Eiszeit den doch terrestrisch-endogenen Oro-

${ }^{33}$ Neben zahlreichen älteren Versuchen sei erwähnt der von $\mathrm{Z}$ inner ${ }^{15}$, S. 90, der ein novaähnliches Stadium der Sonne von dem sog. P.-Cygni-Typus für das Ende der diluvialen Eiszeit heranzog; Fi cke r ${ }^{2}$ wies besonders auf die Arbeit hin. $\mathrm{Himpel^{13 }}$ dachte sogar an ein U-Geminorum- oder Nova-Stadium der Sonne, in dessen Folge zunächst ein Zusammenbruch des eisfreien Klimatypus und anschließend, unter Mitwirkung der astronomischen Theorie, eine glaziale Epoche folgen sollte. Aber der Novazyklus ist, wie in Z. Naturforschg. 1, 414 [1946] gezeigt, nur von der Größenordnung von Jahrzehnten bis Jahrhunderten, während er genesen folge, ist damit allerdings noch nicht gelöst. Wer aber die große Anzahl von Dunkelwolken im Milchstraßensystem berücksichtigt, wird die Möglichkeit, daß die Sonne öfters in einer solchen stand, nicht von der Hand weisen. $\mathrm{Ob}$ eine große Eiszeit oder nur eine Niederschlagsvermehrung mit Lokalvergletscherungen die Folge sein wird, das dürfte auch vom Erdbild mitbestimmt werden. Es könnte sein, daß ein gewisses Ausmaß von Geokratie eine der Bedingungen hierzu wäre. Ein endgültiges Urteil sei aber nicht gegeben, und man sollte sich besser dem abschließenden Urteil S i m p s o n s $^{2}$ anschließen, daß es erforderlich sei, erst eine gesicherte Theorie des Quartärs zu entwickeln, bevor man die früheren Zeitalter in Angriff nimmt!

Nachtrag: Von geologischer Seite bin ich auf die Notwendigkeit folgender Bemerkung zur astronomischen Theorie hingewiesen worden: Es ist klar, daß die Erdbahnexzentrizität nicht nur die Jahressumme; sondern, entgegen weitverbreitetem Irrglauben, auch die Jahreszeiten unbeeinflußt läßt. Der Astronom bezeichnet als Sommerhalbjahr die Zeit, in der die Somne nördlich vom Äquator steht. Der Perihelsommer bei extremer Exzentrizität dauert z. B. vom 1. April bis 14. September, der entsprechende Aphelsommer vom 11. März bis 2. Oktober. Die Gesamtstrahlung in diesen beiden Sommern ist genau die gleiche (!). Zu den sog. „unternormalen“ Sommerbestrahlungen, die nichts als eine reine Fiktion sind, kommt man erst, wenn man als „Sommerhalbjahr“ 182,5 Tage ansetzt - was astronomisch sinnlos ist, woraus die Problematik der ganzen Theorie hervorgeht.

In seinen bekannten Arbeiten (W. S o e r ge l : „Die Vereisungskurve“, Berlin 1937: „Das diluviale System“, Fortschr. Geol. Paläontol. 12, 155 [1939]; Beiträge Geol. Thüringen 5, 269 [1940]) wird der Versuch gemacht, die geologische Wirklichkeit, wie sie uns die Vereisungskurve liefert, mit dem vielzackigen Schema der Strahlungstheorie zu vereinbaren; trotz der hervorragenden Bearbeitung des umfangreichen Beobachtungsmaterials kann man daraus natürlich keinen stärkeren Beweis für die astronomische Theorie herauslesen, als aus älteren (z. B. dem Penck-Brücknerschen der Alpen) und neueren (z. B. dem von Eberl für das Alpenvorland) Eiszeit-

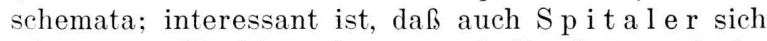
mit seiner Strahlungskurve auf die Soergelsche Vereisungskurve beruft. - Um es noch einmal zu betonen: ein wirkliches experimentum crucis für die

in dieser Hypothese zu $10^{8}$ Jahren angesetzt werden müßte. Außerdem unterliegen alle diese und ähnliche Versuche dem Einwand, erst postuliert werden zu müssen, was bei der oben vorgetragenen Hypothese von Shapley wegfällt. - Einen ersten Hinweis brachte Himpel in ,Himmelswelt“ 1942, Hefte 1/2, 5/6, 11/12, bes. S. 112. Ferner in Wiener Vorträgen (Mitt. geol. Ges. Wien 25, $293 \mathrm{ff}$. [1942]); eine Veröffentlichung befindet sich in den Mitt. Geogr. Ges. Wien im Druck. Einen Hinweis auf die neuen Ergebnisse bringt Prey im Jahresber. Sternw. Wien 1943 in Vierteljahresschrift d. Astron. Ges. 1944, S. 74 u. 77. - Vergl. Fußnote ${ }^{25}$ 
astronomische Theorie an sich wäre der Nachweis, daß die Südhalbkugel während der nördlichen Eiszeit, insbesondere des letzten Würm-Vorstoßes, ein wärmeres Klima gehabt hätte; dies folgt nicht nur zwangsläufig aus der Theorie, sondern auch aus geometeorologischen Uberlegungen. - Eine spezielle Variante der astronomischen Theorie, d. h. das Namhaftmachen einer bestimmten Elementenkombination für klimatologische Konsequenzen, kann nur geprüft werden, wenn die Zuverlässigkeit der absoluten geologischen
Altersbestimmung der diluvialen Phasen erheblich größer ist als bis jetzt. Daß die Würm-Eiszeit, innerhalb der derzeitigen Beobachtungsgenauigkeit, gleichzeitig auf der ganzen Erde war, spricht gegen die astronomische Theorie.

Es ist mir ein Bedürfnis, meinem Heidelberger geologischen Kollegen Prof. Becksmann für reiche wissenschaftliche Beratung und Unterstiutzung von Herzen zu danken.

\section{NOTIZEN}

\section{Uber emanierende Uranverbindungen}

Von $\mathrm{Hans}$ Götte und Gerhard Radoch Kaiser-Wilhelm-Institut für Chemie, Tailfingen (Z. Naturforschg. 2 a, 427 [1947]; eingegangen am 4. August 1947)

Um die bei der Uranspaltung auftretenden Folgeprodukte der Edelgase zu gewinnen, bedient man sich, wie $\mathrm{Hahn}$ und $\mathrm{Str}$ a $\beta$ an $\mathrm{n}$ gezeigt haben, „emanierender“ Uranpräparate ${ }^{1}$. Für die Ausbeute des Verfahrens ist das Emaniervermögen der bestrahlten Präparate ausschlaggebend. Daher sind die Eigenschaften von Uranverbindungen in bezug auf ihr Emaniervermögen von Interesse.

Als oberflächenreiche und daher durch Emanierfähigkeit ausgezeichnete Uranverbindung kommt in erster Linie das Ammoniumuranat in Frage. Die Art der Herstellung ist nicht ohne Einfluß auf das Emaniervermögen. Schnell oder langsam bei Siede- oder Zimmertemperatur ausgeschiedene Präparate zeigen Unterschiede in der Emanationsabgabe. Jedoch sind die Ergebnisse nicht immer reproduzierbar. Einen. wesentlich größeren Einfluß hat die Luftfeuchtigkeit auf das Emaniervermögen des Ammoniumuranats. In mit $\mathrm{CaCl}_{2}$ getrockneter Luft zeigte ein Ammoniumuranat-Präparat 18,3\% Emaniervermögen. Bei $90 \%$ Luftfeuchtigkeit stieg das Emaniervermögen auf 60 bis 70\%. Dieser Prozeß ist reversibel. Bei Überleiten trockener Luft sinkt die Emanierfähigkeit wieder ab.

In Anlehnung an die Versuche von $\mathrm{St} \mathrm{r}$ a $\& \mathrm{~m}$ a $\mathrm{n} \mathrm{n}^{2}$ über das Emaniervermögen organischer Bariumsalze wurde versucht, auch Uranverbindungen mit organischen Gruppen im Molekül herzustellen, um auf diese Weise das Emaniervermögen zu erhöhen. Dazu wurden Uransalzlösungen mit verschiedenen organischen Aminen, wie Methylamin, Diäthylamin, $n$-Butylamin, Isobutylamin, Aminocyclohexylamin, Trimethylamin und Triäthylamin gefällt. Die sich ausscheidenden gelbgefärbten Substanzen waren durch Dekantieren nicht zu reinigen, da sie sehr bald kolloid in Lösung gingen. Die abzentrifugierten und getrockneten Niederschläge stellen aller Wahrscheinlichkeit nach keine definierten Substanzen dar. Die Emanierfähigkeit dieser Verbindungen liegt im allgemeinen höher als die des Ammoniumuranats und erreicht in einigen Fällen in 90 -proz. Luftfeuchtigkeit bis zu $100 \%$ (Iso- butylamin- und Triäthylamin-Ammoniumuranat). Der Feuchtigkeitseinfluß der Luft ist hier noch ausgeprägter. In trockener Atmosphäre sinkt das Emaniervermögen dieser Verbindungen in einzelnen Fällen bis auf 4 bis $8 \%$ ab.

1 O. Hahn u. F. Stra § mann, Naturwiss. 28, 54 [1940].

${ }^{2}$ F. Stra \& mann, Z. physik. Chem. Abt. B 26, $362[1934]$.

\section{Über die Struktur des $\alpha$-Keratins}

Von $\mathrm{Helmut} \mathrm{Z}$ ahn

(Zusammenfassung der in Z. Naturforschg. 2 b, Heft 3/4, S. 104-108 erscheinenden Arbeit)

Eine Diskussion von Konstellationen der Peptidkette zeigt die Unwahrscheinlichkeit ebener Faltungsformen, wenn die Peptidkette nur wenig Glykokoll enthält und bei einem Faltungs-Entfaltungs-Mechanismus die Seitenkettenreste nicht umgeklappt werden dürfen. Durch Rotation der Peptidbindungen wird eine räumliche Faltungsform der Peptidkette konstruiert, welche mit den Röntgendaten des $\alpha$-Keratins verträglich ist. Diese Konstellation erlaubt die Entfaltung zur gestreckten Zickzack-Kette (Verlängerung um 30\%), ohne Rhythmus und Richtung der Seitenketten in bezug auf die Backbone-Ebene zu ändern.

\section{Ǔber die Energieentwertung in Molekülen mit langer Kohlenstoffkette}

Von Fritz B andow*

(Z. Naturforschg. 2a, 427 [1947]; eingeg. am 16. Juni 1947)

Es wird auf eine Beziehung zwischen zwei verschiedenen Versuchsreihen hingewiesen, die für die allgemein wichtige Frage der Energiewanderung und ihrer Störung große Bedeutung hat: die Ausbeute der Fluoreszenzerregung und der photochemischen cistrans-Umwandlung nimmt mit wachsender Zahl der Doppelbindungen ab. In den Molekülen mit langer Kohlenstoffkette tritt also eine Energieentwertung ein.

* Mannheim, Max-Joseph-Str. 7.
Dieses Werk wurde im Jahr 2013 vom Verlag Zeitschrift für Naturforschung in Zusammenarbeit mit der Max-Planck-Gesellschaft zur Förderung der Wissenschaften e.V. digitalisiert und unter folgender Lizenz veröffentlicht: Creative Commons Namensnennung-Keine Bearbeitung 3.0 Deutschland Lizenz.

Zum 01.01.2015 ist eine Anpassung der Lizenzbedingungen (Entfall der Creative Commons Lizenzbedingung „Keine Bearbeitung“) beabsichtigt, um eine Nachnutzung auch im Rahmen zukünftiger wissenschaftlicher Nutzungsformen zu ermöglichen.
This work has been digitalized and published in 2013 by Verlag Zeitschrift für Naturforschung in cooperation with the Max Planck Society for the Advancement of Science under a Creative Commons Attribution-NoDerivs 3.0 Germany License.

On 01.01.2015 it is planned to change the License Conditions (the removal of the Creative Commons License condition "no derivative works"). This is to allow reuse in the area of future scientific usage. 